\title{
Reduced recruitment of orbitofrontal cortex to human social chemosensory cues in social anxiety
}

\author{
Wen Zhou ${ }^{a}$, Ping Hou ${ }^{\mathrm{b}, \mathrm{c}}$, Yuxiang Zhou ${ }^{\mathrm{b}}$, Denise Chen ${ }^{\mathrm{d}, *}$ \\ a Key Laboratory of Mental Health, Institute of Psychology, Chinese Academy of Sciences, 4A Datun Road, Chaoyang District, Beijing 100101, China \\ b Department of Diagnostic and Interventional Imaging, University of Texas Medical School in Houston, 6431 Fannin Street, MSB 2.130B, Houston, TX 77030, USA \\ c Department of Imaging Physics, UT M. D. Anderson Cancer Center, 1515 Holcombe Boulevard, Unit 56, Houston, TX 77030-4009, USA \\ d Department of Psychology MS-25, Rice University, 6100 Main Street, Houston, TX 77005, USA
}

\section{A R T I C L E I N F O}

\section{Article history:}

Received 19 August 2010

Revised 13 November 2010

Accepted 23 December 2010

Available online 30 December 2010

\begin{abstract}
A B S T R A C T
Social anxiety refers to the prevalent and debilitating experience of fear and anxiety of being scrutinized in social situations. It originates from both learned (e.g. adverse social conditioning) and innate (e.g. shyness) factors. Research on social anxiety has traditionally focused on negative emotions induced by visual and auditory social cues in socially anxious clinical populations, and posits a dysfunctional orbitofrontal-amygdala circuit as a primary etiological mechanism. Yet as a trait, social anxiety is independent of one's specific emotional state. Here we probe the neural substrate of intrinsic social anxiety by employing a unique type of social stimuli, airborne human social chemosensory cues that are inherently social, ubiquitously present, and yet operating below verbal awareness. We show that the adopted social chemosensory cues were not perceived to be human-related, did not differentially bias self-report of anxiety or autonomic nervous system responses, yet individuals with elevated social anxiety demonstrated a reduced recruitment of the orbitofrontal cortex to social chemosensory cues. No reciprocal activity in the amygdala was observed. Our findings point to an intrinsic neural substrate underlying social anxiety that is not associated with prior adverse social conditioning, thereby providing the first neural evidence for the inherent social aspect of this enigmatic phenomenon.
\end{abstract}

(C) 2011 Elsevier Inc. All rights reserved.

\section{Introduction}

Social anxiety, described since the time of Hippocrates [as quoted in (Marks, 1969)], is the most common anxiety disorder with a lifetime prevalence of 13\% (Kessler et al., 1994); yet its underlying mechanism remains little understood. Existing studies on social anxiety are mostly based on the clinical population: individuals who experience excessive anxiety in social situations that interfere with their daily routine [e.g. (Beidel et al., 1985; Fedoroff and Taylor, 2001; Gelernter et al., 1991; Liebowitz et al., 1985)]. According to these studies, adverse social conditioning is a primary factor for social anxiety. When placed in anxiety-provoking social situations, people with clinical social anxiety report experiencing recurrent negative and distorted images (visual, body sensational, auditory, but rarely olfactory) which are linked to memories of adverse social events that clustered around the onset of the disorder (Hackmann et al., 2000). Furthermore, these patients can be treated with exposure interventions, which involve unconditioning, i.e., decoupling the connection between a social situation and its feared consequences

\footnotetext{
* Corresponding author. Fax: +1 7133485221.

E-mail address: xdchen@rice.edu (D. Chen).
}

(Gould et al., 1997). However, individual differences have also been observed that cannot be attributed to adverse social experience. There is an increased rate of social anxiety symptoms among the family members of people with clinical social anxiety (Fyer et al., 1993), and the heritability is estimated to be $30 \%-40 \%$ in women (Kendler et al., 1992). Moreover, childhood shyness, a partly genetic trait (Plomin and Daniels, 1986), also contributes to clinical social anxiety (Stemberger et al., 1995). These findings point to an innate mechanism underlying people's proneness to social anxiety, without involving adverse social conditioning from experience.

Current neural models of social anxiety tend to build upon two heavily interconnected regions: the orbitofrontal cortex (OFC) and the amygdala (Davidson, 2002; Goldin et al., 2009; Milad and Rauch, 2007). While the amygdala is well documented to respond to conditioned fear (Davis, 1992), the OFC is well documented to underlie social behavior including emotion, stimulus-reinforcement association and reversal, and decision-making. Specifically, medial orbitofrontal cortex is implicated in the monitoring, learning and memory of the reward value of reinforcers, and lateral orbitofrontal cortex is related to the evaluation of punishers that can lead to a change in behavior (Kringelbach and Rolls, 2004). Patients with damage to the orbitofrontal cortex are impaired in correctly identifying social signals including face and voice expressions (Hornak 
et al., 1996), among other symptoms. Accordingly, exaggerated amygdala activity along with the dysfunction of the OFC during exposure to social settings is predicted to give rise to the corresponding fearful experience. This hypothesis is supported by brain imaging data showing enhanced amygdala activity and reduced medial orbitofrontal response during public speaking (Tillfors et al., 2001), as well as positive association between the amygdala and lateral orbitofrontal activation when anticipating peer evaluation (Guyer et al., 2008) in individuals with clinical social anxiety relative to normal controls. It is also consistent with the observations that social anxiety is related to previous conditioning of adverse social events. Little, however, is known about the neural underpinnings of the nonadverse conditioning components of social anxiety.

In this work, we address this issue by eliminating the adverse conditioning component in the social anxiety circuitry. We have done so by employing a unique kind of social signal, the chemosensory cues of natural human sweat. Sweat has been shown to convey social information including individual identity (Russell, 1976), reproductive state (Stern and McClintock, 1998), and affect (happiness, fear, anxiety) of the sweat donors (Ackerl et al., 2002; Chen and HavilandJones, 2000; Chen et al., 2006; Mujica-Parodi et al., 2009; Pause et al., 2004). Yet unlike visual or auditory social cues, regular sweat is not known to induce subjective stressful or fearful feelings (Zhou and Chen, 2009). Furthermore, as demonstrated in our recent study in a different context (Zhou and Chen, 2008), the socio-emotional aspect of human sweat is encoded in the OFC, a structure documented to process social information (Adolphs, 2003), despite the fact that subjects were verbally unaware of the nature of the olfactory stimuli. Taking advantage of this finding, we have in the present study assessed the social anxiety levels in the same subjects, conducted a new study assessing physiological responses to chemosensory social cues, and analyzed the fMRI data for the particular purpose of identifying the neural correlates of individual differences in social anxiety.

We predict that if orbitofrontal dysfunction is dependent on exaggerated amygdala activity, no correlation between social anxiety and OFC response should be seen in the absence of amygdala activation. Alternatively, if there is inherent individual variation in social signal processing independent of adverse conditioning, as in line with the findings from genetic studies (Fyer et al., 1993; Kendler et al., 1992; Stemberger et al., 1995), a significant correlation is expected between social anxiety and orbitofrontal response to social chemosensory cues.

\section{Materials and methods}

\section{Participants}

The study was approved by the institutional review board at Rice University and The University of Texas Medical School at Houston. All subjects gave written informed consent for participation. Only men were recruited as sweat donors because the apocrine glands in the underarm are larger in men than in women (Doty, 1981). Only women were recruited as odor recipients because of their superior sense of smell (Brand and Millot, 2001) and sensitivity to emotional signals (Brody and Hall, 2000).

Six healthy male non-smokers (mean age $=27.3$ years, $\mathrm{SE}=1.03$ ) from a larger study were chosen as sweat donors (see Sweat selection and preparation). Three of these men served as sweat donors for the fMRI experiment, and three served as sweat donors for the experiment on autonomic nervous system response to the olfactory stimuli.

Nineteen right-handed healthy females (Mean age $=23.4$ years; $\mathrm{SE}=0.93$ years) with varying degrees of social anxiety (mean $=14$, $\mathrm{SE}=1.17$, see Social anxiety assessment) were selected from a panel of over 40 volunteers to participate in the fMRI experiment as odor recipients. We choose to study healthy normal population because of our interest in the inherent neural correlate of individual propensity to social anxiety. Subjects reported to have no rhinal disorders or psychological problems or neurological diseases and showed superior olfactory sensitivity to phenyl ethyl alcohol (PEA), a nonsocial rose like odor $[$ Mean $=0.00046 \%$, SE in binary dilution step $=0.63$; in comparison to Mean $=0.05 \%$ as reported in (Tsukatani et al., 2003)], and androstadienone (ANDR), the putative human sex pheromone (Jacob et al., 2001; Savic et al., 2001, 2005) [Mean $=2.9 \mu \mathrm{M}, \mathrm{SE}$ in binary dilution step $=0.51$; in comparison to Mean $=211 \mu \mathrm{M}$ as reported in (Lundström et al., 2003b)]. Threshold of phenyl ethyl alcohol (PEA) and androstadienone, both diluted in propylene glycol in binary dilution steps, were assessed using a triple-forced-choice ascending staircase with reversal design (Hummel et al., 1997). PEA was presented with Sniffin' Sticks (Burghart Medical Technology, Wedel, Germany). Androstadienone was presented on nylon/polyester blended pads in petri dishes. Subjects were presented with 3 sticks/petri dishes-one containing the target smell, the other two the diluent-and asked to identify the target. They were presented with a lower concentration if they had made 2 consecutively correct identifications, and a higher concentration immediately after they made a single error. Threshold was calculated base on the average of the last four reversals.

To verify that sweat does not induce anxiety, we tested another group of fifteen healthy female subjects (Mean age $=23.7$ years; $\mathrm{SE}=0.97$ ) with good olfactory sensitivities (for each subject, $\mathrm{PEA}<0.016 \%, \mathrm{ANDR}<916 \mu \mathrm{M})$ and similar social anxiety scores as the fMRI subjects ( mean $=12.7, \mathrm{SE}=1.08$ ). They performed the same paradigm used in the fMRI experiment (see Procedure) while their autonomic nervous system responses (heart rate and skin conductance, see Physiological recordings) and self-reported mood (happy, sad, fearful, aroused) were monitored.

None of the female subjects were on hormone contraceptives. The subjects were tested during the periovulatory phase of their menstrual cycles (10-27 days, on average 18 days, from the onset of their last period in the fMRI experiment; 7-29 days, on average 17 days, from the onset of their last period in the physiological recording experiment). They were informed that the study was on brain activations/autonomic responses to natural compounds and were blind to the nature of the olfactory stimuli used in the experiment.

\section{Sweat selection and preparation}

As mentioned above, donors were selected from a larger study with 20 heterosexual male donors. They refrained from using deodorant/antiperspirant/scented products, and used scent-free shampoo/conditioner, soap, and lotion provided by the experimenter from two days prior to the sweat collection experiment until the end of the experiment. Two types of sweat were collected, neutral sweat, encountered in daily social contact; and sexual sweat, typically encountered in intimate relationships. The emotions of neutrality and sexual arousal were induced with 20 -minute-long video segments showing educational documentaries and sexual intercourse between heterosexual couples, respectively. The donors kept a $4^{\prime \prime} \times 4^{\prime \prime}$ pad (rayon/polyester for maximum absorbance) in each armpit while watching the video segments. Meanwhile, their skin conductance was recorded using $\mathrm{Ag} / \mathrm{AgCl}$ electrodes filled with isotonic electrolyte (BIOPAC Systems, Inc., Goleta, CA) attached to the palmar area of the non-dominant hand. New pads were used for each segment. The sequence of segment presentation was counterbalanced. A 5 min segment (of the same emotional content as the 20 min segment) preceded each 20 min segment, serving as an emotional transition. Only pads worn during the $20 \mathrm{~min}$ segments were used. Once collected, sweat pads were stored at $-80^{\circ} \mathrm{C}$ until subsequent testing. Over the course of the 20 min video segments donors experienced 
greater arousal (measured by skin conductance) while watching erotic videos as compared with watching neutral videos $(\mathrm{p}=0.017)$.

Six healthy, male non-smokers were subsequently selected for the current study mainly because of their high levels of the self-reported sexual arousal and neutrality during the sweat collection (see Supplementary Figure S1 for their self-reported emotional response to the videos).

\section{Olfactory stimuli}

Two types of human sweat were used: the pooled sweat of sexual arousal obtained from 3 male donors (sexual sweat), and the sweat from the same men but under neutral emotions (neutral sweat). In addition, we included the putative sex pheromone androstadienone (ANDR: Steraloids Inc. Newport, RI; $916 \mu \mathrm{M}$ in propylene glycol, $1 \mathrm{ml}$ ) and phenyl ethyl alcohol (PEA: Sigma-Aldrich, St. Louis, MO; 0.03\% in propylene glycol, $1 \mathrm{ml}$ ), which served as a nonsocial control. The concentrations of ANDR and PEA were chosen based on a separate panel of 10 female judges who were told to best match their intensity and pleasantness to the sweat samples. All smells were presented on nylon/polyester blended pads.

\section{Social anxiety assessment}

Social anxiety was assessed with the social anxiety subscale of the Self Consciousness Scale (Fenigstein et al., 1975). It consists of six items (e.g. Large groups make me nervous; I have trouble working when someone is watching me) rated on a scale of 0 (extremely uncharacteristic) to 4 (extremely characteristic), and measures the discomfort in the presence of others. It has a reliability of 0.73 and is sensitive to the individual differences in the normal population (Fenigstein et al., 1975). It also correlates well with other social anxiety scales (Leary, 1983).

\section{Procedure}

For the fMRI experiment, each olfactory stimulus was presented twice per run over a course of 5 runs in a semi-randomized manner via a non-ferric, computer-controlled, 6-channel MRI compatible olfactometer (Mindware Technologies LTD., Columbus, $\mathrm{OH}$ ) adapted after Lorig (Lorig et al., 1999). Each olfactory presentation consisted of inhalation (4s), exhalation (4s) and another inhalation (4s) (12 s in total), prompted by "I" (for breath in) and "O" (for breath out) signs on the screen so that breathing can be synchronized with smell delivery (All subjects had been trained to breathe according to the rhythm of the visual prompts prior to the scans). The presentations were interleaved with a $28 \mathrm{~s}$ interval in between, during which only purified air was delivered and subjects followed "I" and "O" prompts as usual. Immediately following each smell presentation, subjects rated either the intensity or the pleasantness of the smell on a 1-5 scale with a response pad attached to their right hand (see Supplementary Figure S2). The order in presenting the intensity and pleasantness questions was counterbalanced across the subjects and the scans. After the scans, 16 out of the 19 subjects smelled the same olfactory stimuli presented through the olfactometer and described what each smelled like. Each fMRI session lasted about $1 \mathrm{~h}$.

The same paradigm was adopted to examine autonomic nervous system responses to the olfactory stimuli, with one exception: after rating the intensity/pleasantness of an odor, subjects also rated on a 100 -unit visual analogue scale how happy, fearful, sad, or aroused they felt.

\section{fMRI data acquisition}

fMRI Data were collected using a $3 \mathrm{~T}$ Philips Intera and asymmetric spin echo pulse sequence (Stables et al., 1998) with $\mathrm{TE}=31 \mathrm{~ms}$,
$\mathrm{TR}=2000 \mathrm{~ms}$, FOV $=240 \times 240 \mathrm{~mm}^{2}$, matrix size $=128 \times 128$, and number of slices $=28$. The voxel dimension was $1.875 \times 1.875 \times 3.75 \mathrm{~mm}^{3}$. Four dummy scans were conducted at the beginning of each run to allow the magnetization to reach magnetic equilibrium. A T1 weighted anatomical volume (3D MPRAGE; $1 \times 1 \times 1 \mathrm{~mm}^{3}$ resolution) was also acquired for each subject for normalization and visualization of the functional data. Slices were acquired using oblique orientation $30^{\circ}$ to the AC-PC line (Sobel et al., 1997). Respiration was recorded via MP150 (BIOPAC Systems, Inc., Goleta, CA), connected with the scanner's built-in sensor taped on subjects' diaphragms.

\section{fMRI data analysis}

Functional data were analyzed using AFNI (Cox, 1996). Following image preprocessing, the fMRI data were analyzed using the general linear model. Regressors of interest, each corresponding to the time points when subjects inhaled a particular olfactory stimulus, were convolved with the gamma function. The parameter estimates (betas) for each regressor were calculated for all brain voxels and entered in the second level ANCOVA together with the average intensity and pleasantness ratings for each olfactory stimulus from each subject. The nonsocial control smell (PEA) was set as the baseline. The use of ANCOVA allowed us to normalize the inherent intensity and pleasantness variations (see Olfactory stimuli intensity and pleasantness) in the olfactory stimuli. Alpha probability simulation was used to apply small volume correction to the orbitofrontal cortex (OFC) and the amygdala in the voxel-wise analysis based on their heavy involvement in social anxiety (Davidson, 2002; Milad and Rauch, 2007; Tillfors et al., 2001).

\section{Regions of interest (ROIs)}

The OFC and the amygdala are heavily involved in social anxiety. In keeping with our primary interest in these regions, we defined a functional OFC ROI in the right OFC (Talairach coordinates 33, 40, - 1, cluster size $=52 \mathrm{~mm}^{3}$; see Results) that significantly differentiated sweat from the nonsocial control PEA in the ANCOVA test. The amygdala did not significantly differentiate among the olfactory conditions (see Results). To explore whether there was any connection between social anxiety and amygdala activity, we structurally defined the left amygdala and the right amygdala using (Talairach and Tournoux, 1988), which comes with AFNI as a publicly available structural mask. The average \% signal changes within the ROIs (functionally defined OFC ROI and structurally defined left and right amygdala ROIs) were then extracted for each olfactory condition from each subject and entered in a mixed linear model as the dependant variable, with olfactory condition as the repeated factor, and social anxiety score, intensity and pleasantness ratings as the covariates. The interaction between olfactory condition and social anxiety score, as well as the main effects of olfactory condition, perceived intensity, and perceived pleasantness, was assessed in the model. In addition, partial correlation tests were performed between social anxiety scores and the \% signal changes within the ROIs, controlling for intensity/pleasantness ratings. This is because the amygdala and the OFC, being primary and secondary olfactory regions, respectively, are known to process olfactory intensity and pleasantness (Anderson et al., 2003; Gottfried and Zald, 2005).

\section{Physiological recordings}

Heart rate and skin conductance were recorded using Biopac MP150 (Goleta, CA). Electrocardiogram signals were recorded using disposable snap electrodes attached to the right collarbone and the left and right (ground) rib cage. Skin conductance was recorded using an $8-\mathrm{mm}$ in diameter $\mathrm{Ag} / \mathrm{AgCl}$ electrodes filled with Biopac isotonic 
electrode paste and attached bipolarly to the palmar area of the nondominant hand. Conductance signals were measured in terms of mean skin conductance amplitude in microsiemens. Data were analyzed using Biopac Acknowledge 3.7.3 and mixed linear model, controlling for variances in the perceived intensity and pleasantness of the olfactory stimuli (see Olfactory stimuli intensity and pleasantness).

\section{Results}

Olfactory stimuli intensity and pleasantness

The four olfactory stimuli (sexual sweat, neutral sweat, PEA, and ANDR) were all rated as supra-threshold in both the fMRI and the physiological recording experiments $(\mathrm{ps}<0.001$ ). Overall, subjects who participated in the fMRI experiment perceived the sexual sweat and ANDR to be more intense than the neutral sweat ( $p=0.019$ and $\mathrm{p}<0.001$, respectively, Bonferroni corrected); and the PEA to be more pleasant than both the sexual sweat and neutral sweat $(\mathrm{p}=0.001$ and
0.019, respectively, Bonferroni corrected). Subjects in the physiological recording experiment perceived ANDR to be less intense than sexual sweat and PEA $(p<0.001$ and $p=0.01$, respectively, Bonferroni corrected) and more pleasant than neutral sweat and sexual sweat $(\mathrm{p}=0.046$ and $\mathrm{p}<0.001$, respectively, Bonferroni corrected). They perceived sexual sweat to be more intense than neutral sweat $(p=0.024$, Bonferroni corrected). These perceptual differences were statistically controlled for in the subsequent analyses of the subjects' mood ratings, physiological responses, and brain activities (See Materials and methods).

\section{Social chemosensory cues and anxiety}

None of the subjects verbally reported experiencing anxiety throughout the fMRI session or the physiological recording session. Compared with the nonsocial control PEA, human-related chemosensory cues (sexual sweat, neutral sweat, and ANDR) did not differentially change self-reported mood $(\mathrm{p}=0.40,0.72,0.28$, and 0.52 for happiness,
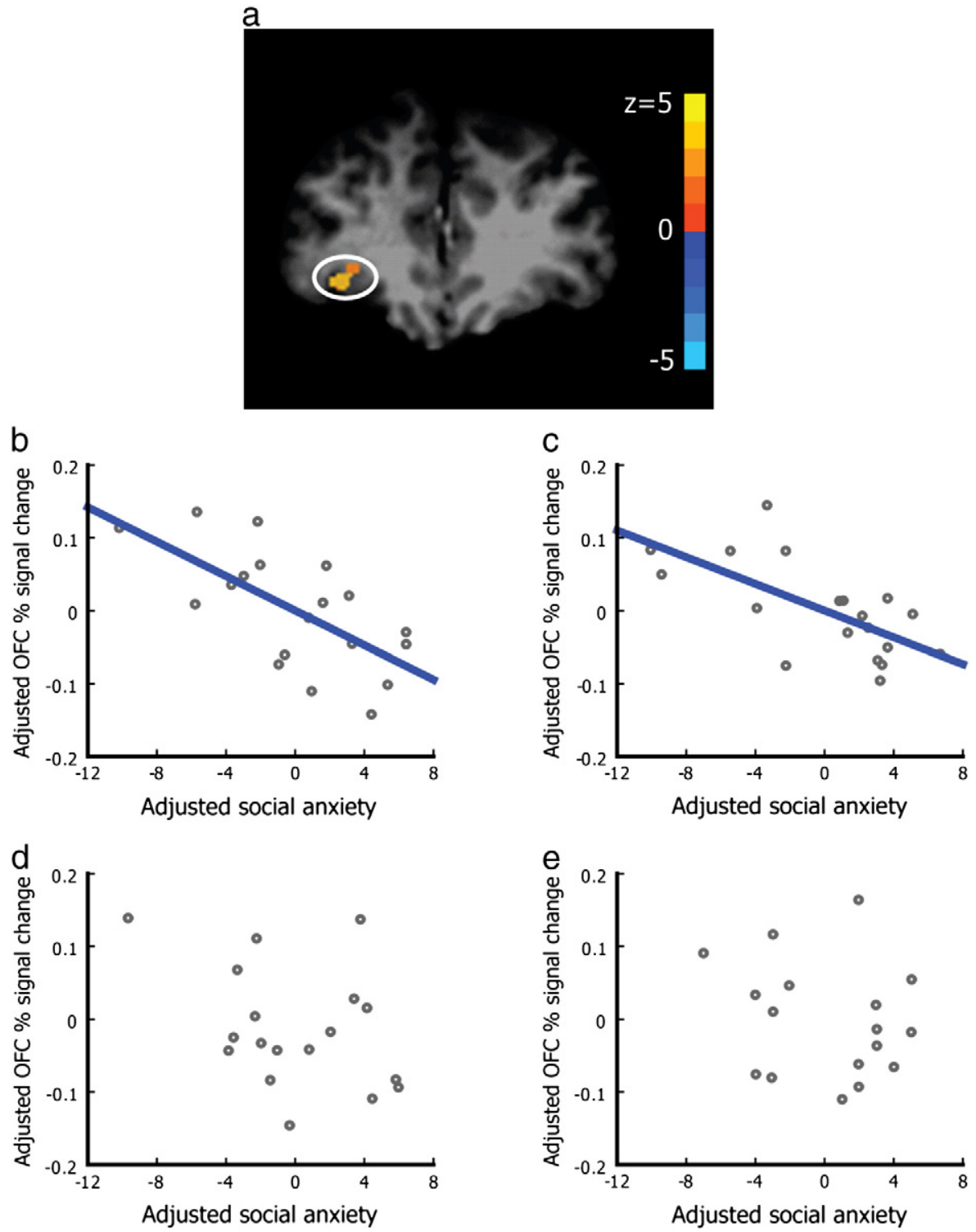

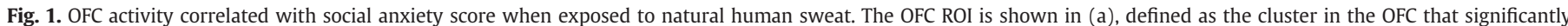

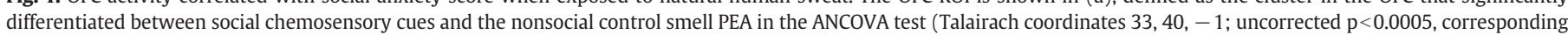

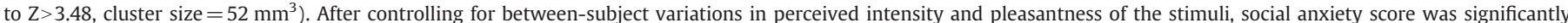

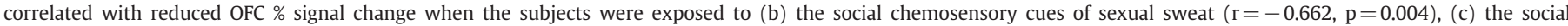

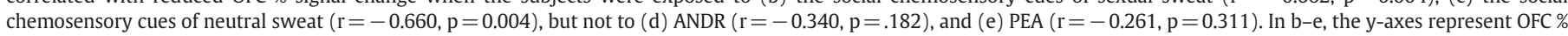

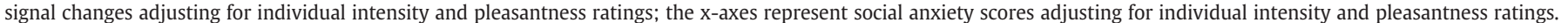


fear, sadness, and arousal, respectively) or autonomic nervous system responses $(p=0.19$ and 0.20 for heart rate and skin conductance, respectively). There was no correlation between social anxiety and the mood ratings ( $\mathrm{ps}>0.40$, Bonferroni corrected) or the physiological measures ( $p s>0.42$, Bonferroni corrected) when subjects were exposed to any of these chemosensory cues. In addition, social anxiety did not significantly affect the perceived intensity and pleasantness ratings ( $\mathrm{ps}>0.12$, Bonferroni corrected, in the fMRI experiment; ps $>0.27$, Bonferroni corrected, in the physiological recording experiment) of the olfactory stimuli. Finally, there was no difference in either the breathing amplitude $(p=0.602)$ or the frequency $(p=0.905)$ across the four olfactory conditions in the fMRI session.

OFC but not amygdala differentiated between social chemosensory cues and nonsocial smell

The ANCOVA test revealed a region in the right OFC (Talairach coordinates $33,40,-1$; uncorrected $\mathrm{p}<0.0005$, corresponding to $\mathrm{Z}>3.48$, cluster size $=52 \mathrm{~mm}^{3}$, svc corrected $\mathrm{P}<0.005$, peak $\mathrm{Z}=3.90$; Fig. 1a) that distinguished among the four olfactory stimuli. Post hoc tests showed this region significantly responded to the sexual sweat as compared with the nonsocial baseline, and to the sexual sweat as compared with either the neutral sweat or ANDR ( $P<0.001$, Bonferroni corrected). As a preparatory step for further analyses, this enabled us to functionally define the OFC ROI whose \% signal changes are correlated with each individual's social anxiety level below (see Materials and methods). A detailed description of the brain responses to the socio-emotional contents of human chemosensory cues has been reported elsewhere (Zhou and Chen, 2008). The focus here is the inherent neural correlates of individual propensity to social anxiety. Consistent with the lack of subjective anxiety report, the amygdala failed to differentiate between any of the sexual sweat, neutral sweat, or ANDR from the nonsocial baseline (PEA) even at a very liberal amplitude threshold of 0.01 (svc corrected $\mathrm{p}>0.6$ ).

\section{Social anxiety and OFC reactivity}

Mixed linear model analysis revealed a significant interaction between olfactory condition and social anxiety score $[F(4,18.13)$ $=4.49, \mathrm{p}=0.011]$ in the right OFC. After controlling for betweensubject variations in the perceived intensity and pleasantness of the stimuli, significant negative correlations were observed between subjects' social anxiety scores and the OFC (Talairach coordinates 33 , $40,-1 ; 52 \mathrm{~mm}^{3}$, see the section above and Regions of interest) \% signal changes during exposures to the sexual sweat $(r=-0.662$, $\mathrm{p}=0.004$, Fig. $1 \mathrm{~b})$ and the neutral sweat $(\mathrm{r}=-0.660, \mathrm{p}=0.004$, Fig. 1c). No such correlation was observed for ANDR ( $\mathrm{r}=-0.340$, $\mathrm{p}=.182$, Fig. $1 \mathrm{~d})$ or PEA $(\mathrm{r}=-0.261, \mathrm{p}=0.311$, Fig. $1 \mathrm{e})$.

\section{Social anxiety and amygdala reactivity}

Different from the OFC, the \% signal changes in both the left and the right amygdala (structurally defined, see Regions of interest) were not correlated with social anxiety scores during the exposure to any of the olfactory stimuli (controlling for intensity and pleasantness ratings, ps $>0.33$ ). Nor was there any interaction between olfactory condition and social anxiety score $[F(4,16.47)=0.127, p=0.97$ for the left amygdala; $\mathrm{F}(4,18.05)=1.886, \mathrm{p}=0.16$ for the right amygdala].

\section{Discussion}

The primary purpose of the current study is to probe the neural substrate of intrinsic social anxiety using human social chemosensory cues that are inherently social yet operate below verbal awareness. For reasons discussed earlier, we used only male sweat donors and female odor recipients. We are therefore not in a position to examine gender differences in brain response patterns to these chemosensory cues. Future studies will elucidate whether such responses (e.g. brain response to sexual sweat) are sexually dimorphic.

In women, ANDR is distinguished from common household smells in terms of neural responses (Savic et al., 2001, 2005). It exerts a positive effect on mood and cognition (Bensafi et al., 2004; Jacob and McClintock, 2000; Lundström et al., 2003a; Wyart et al., 2007) and heightens sympathetic nervous system arousal (Bensafi et al., 2004; Jacob et al., 2001; Wyart et al., 2007) in a dose (Bensafi et al., 2004) and context (Jacob et al., 2001; Lundström and Olsson, 2005) dependent manner. In the current study, the subjects smelled a low dose of ANDR $(916 \mu \mathrm{M})$ via the olfactometer (hence with little context), which likely contributes to the lack of significant difference in the mood ratings and physiological responses between the conditions of ANDR and PEA. Although ANDR is considered a meaningful chemosignal, the OFC response to ANDR did not significantly correlate with social anxiety. This is probably because ANDR acts more on sex/reproduction related regions (Savic et al., 2001, 2005), and does not carry rich social information in and of itself, as it is one of the many compounds that comprise natural sweat (Gower et al., 1994; Penn et al., 2007).

Although the amplitude of the OFC response to the sexual sweat was significantly larger than that of the neutral sweat, even after controlling for their perceived intensity and pleasantness differences, the correlations between social anxiety scores and the OFC responses to these two types of social chemosensory cues were strikingly similar. In other words, although the emotional content of sexual sweat augmented OFC responses in general, as one would expect based on the involvement of OFC in social emotional processing (Hornak et al., 1996; Kringelbach and Rolls, 2004), there is an invariant component in the OFC's responsiveness to these social signals.

As a trait, social anxiety is independent of one's specific emotional state. The adopted social chemosensory cues of natural sweat did not trigger amygdala responses [See (Mujica-Parodi et al., 2009) for the case of fearful sweat], subjective anxious/fearful feelings, or autonomic nervous system responses. This is corroborated by the independence of amygdala activity and social anxiety scores during the exposures to these social cues. Yet subjects with higher anxiety scores showed inherently reduced orbitofrontal responses to the sweat of neutrality (encountered in daily social contact), and to the sweat of sexual arousal (typically encountered in intimate relationships). This, however, is not true in response to either the nonsocial control PEA or the human steroid ANDR. The observed pattern of the neural responses to the two human sweats is especially interesting in light of the subjects' unawareness of the nature of the olfactory stimuli; indeed, the majority (nearly 90\%) of the subjects did not consciously associate the social chemosensory cues with humans (Zhou and Chen, 2008). This implies that humans, as an intensely social species, process social signals in an automatic and sometimes subconscious manner.

Taken together, by utilizing the variance of social anxiety levels in the normal population, our results go beyond previous patients' data and demonstrate a linkage between social anxiety and reduced orbitofrontal response to natural human social chemosensory cues in the absence of any anxiety experience. No such linkage was found between social anxiety and amygdala activity. Such functional dissociation is in line with recent evidence that the OFC and the amygdala assume different roles in general anxiety (Bishop, 2009), as well as the documented role of OFC in social processing (Adolphs, 2003). Our findings provide direct neural support for the hypothesis raised earlier, viz. there are inherent variations in the orbitofrontal responsiveness to social signals. These variations are linked to social anxiety, but are independent of anxiety experience or adverse social conditioning. Whether such inherent variations can be directly mapped onto genetic differences or personality traits in both normal and clinical populations, is an important open question and this deserves serious studies in the future. 


\section{Acknowledgments}

This work was supported by NIH R03 DC4956. The purchase of the MRI scanner was partially funded by NCRR/NIH grant \# S10RR19186-01. We thank Vips Patel for the assistance with fMRI data collection, and Jay Gottfried, Wen Li, Gang Chen, Michael S. Beauchamp for helpful discussions.

\section{References}

Ackerl, K., Atzmueller, M., Grammer, K., 2002. The scent of fear. Neuro Endocrinol. Lett. 23, 79-84.

Adolphs, R., 2003. Cognitive neuroscience of human social behaviour. Nat. Rev. Neurosci. 4, 165-178.

Anderson, A.K., Christoff, K., Stappen, I., Panitz, D., Ghahremani, D.G., Glover, G., Gabrieli, J.D., Sobel, N., 2003. Dissociated neural representations of intensity and valence in human olfaction. Nat. Neurosci. 6, 196-202.

Beidel, D.C., Turner, S.M., Dancu, C.V., 1985. Physiological, cognitive and behavioral aspects of social anxiety. Behav. Res. Ther. 23, 109-117.

Bensafi, M., Tsutsui, T., Khan, R., Levenson, R.W., Sobel, N., 2004. Sniffing a human sexsteroid derived compound affects mood and autonomic arousal in a dosedependent manner. Psychoneuroendocrinology 29, 1290-1299.

Bishop, S.J., 2009. Trait anxiety and impoverished prefrontal control of attention. Nat. Neurosci. 12, 92-98.

Brand, G., Millot, J.L., 2001. Sex differences in human olfaction: between evidence and enigma. Q. J. Exp. Psychol. B 54, 259-270.

Brody, L.R., Hall, J.A., 2000. Gender, emotion, and expression. In: Lewis, M., HavilandJones, J. (Eds.), Handbook of emotions. Guilford Press, New York, pp. 338-349.

Chen, D., Haviland-Jones, J., 2000. Human olfactory communication of emotion. Percept. Mot. Skills 91, 771-781.

Chen, D., Katdare, A., Lucas, N., 2006. Chemosignals of fear enhance cognitive performance in humans. Chem. Senses 31, 415-423.

Cox, R.W., 1996. AFNI: software for analysis and visualization of functional magnetic resonance neuroimages. Comput. Biomed. Res. 29, 162-173.

Davidson, R.J., 2002. Anxiety and affective style: role of prefrontal cortex and amygdala. Biol. Psychiatry 51, 68-80.

Davis, M., 1992. The role of the amygdala in fear and anxiety. Annu. Rev. Neurosci. 15, 353-375.

Doty, R.L., 1981. Olfactory communication in humans. Chem. Senses 6, 351-376.

Fedoroff, I.C., Taylor, S., 2001. Psychological and pharmacological treatments of social phobia: a meta-analysis. J. Clin. Psychopharmacol. 21, 311-324.

Fenigstein, A., Scheier, M.F., Buss, A.H., 1975. Public and private self-consciousness: assessment and theory. J. Consult. Clin. Psychol. 43, 522-527.

Fyer, A.J., Mannuzza, S., Chapman, T.F., Liebowitz, M.R., Klein, D.F., 1993. A direct interview family study of social phobia. Arch. Gen. Psychiatry 50, 286-293.

Gelernter, C.S., Uhde, T.W., Cimbolic, P., Arnkoff, D.B., Vittone, B.J., Tancer, M.E., Bartko, J.J. 1991. Cognitive-behavioral and pharmacological treatments of social phobia. A controlled study. Arch. Gen. Psychiatry 48, 938-945.

Goldin, P.R., Manber, T., Hakimi, S., Canli, T., Gross, J.J., 2009. Neural bases of social anxiety disorder: emotional reactivity and cognitive regulation during social and physical threat. Arch. Gen. Psychiatry 66, 170-180.

Gottfried, J.A., Zald, D.H., 2005. On the scent of human olfactory orbitofrontal cortex: meta-analysis and comparison to non-human primates. Brain Res. Rev. 50, 287-304.

Gould, R.A., Buckminster, S., Pollack, M.H., Otto, M.W., Massachusetts, L.Y., 1997. Cognitive-behavioral and pharmacological treatment for social phobia: a metaanalysis. Clin. Psychol. Sci. Pr. 4, 291-306.

Gower, D.B. Holland, KT. Mallet, A.J., Rennie, PJ. Watkins, W. 1994 Comparison of 16 androstene steroid concentrations in sterile apocrine sweat and axillary secretions: interconversions of 16-androstenes by the axillary microflora -a mechanism for axillary odour production in man? J. Steroid Biochem. Mol. Biol. 48, 409-418.

Guyer, A.E., Lau, J.Y., Mcclure-Tone, E.B., Parrish, J., Shiffrin, N.D., Reynolds, R.C., Chen, G., Blair, R.J., Leibenluft, E., Fox, N.A., Ernst, M., Pine, D.S., Nelson, E.E., 2008. Amygdala and ventrolateral prefrontal cortex function during anticipated peer evaluation in pediatric social anxiety. Arch. Gen. Psychiatry 65, 1303-1312.

Hackmann, A., Clark, D.M., Mcmanus, F., 2000. Recurrent images and early memories in social phobia. Behav. Res. Ther. 38, 601-610.

Hornak, J., Rolls, E.T., Wade, D., 1996. Face and voice expression identification in patients with emotional and behavioural changes following ventral frontal lobe damage. Neuropsychologia 34, 247-261.

Hummel, T., Sekinger, B., Wolf, S.R., Pauli, E., Kobal, G., 1997. 'Sniffin' sticks': olfactory performance assessed by the combined testing of odour identification, odor discrimination and olfactory threshold. Chem. Senses 22, 39-52.

Jacob, S., Hayreh, D.J.S., Mcclintock, M.K., 2001. Context-dependent effects of steroid chemosignals on human physiology and mood. Physiol. Behav. 74, 15-27.
Jacob, S., Mcclintock, M.K., 2000. Psychological state and mood effects of steroidal chemosignals in women and men. Horm. Behav. 37, 57-78.

Kendler, K.S., Neale, M.C., Kessler, R.C., Heath, A.C., Eaves, L.J., 1992. The genetic epidemiology of phobias in women. The interrelationship of agoraphobia, social phobia, situational phobia, and simple phobia. Arch. Gen. Psychiatry 49, 273-281.

Kessler, R.C., Mcgonagle, K.A., Zhao, S., Nelson, C.B., Hughes, M., Eshleman, S., Wittchen, H.U., Kendler, KS. 1994. Lifetime and 12-month prevalence of DSM-III-R psychiatric disorders in the united states: results from the national comorbidity survey. Arch. Gen. Psychiatry 51, 8-19.

Kringelbach, M.L., Rolls, E.T., 2004. The functional neuroanatomy of the human orbitofrontal cortex: evidence from neuroimaging and neuropsychology. Prog. Neurobiol. 72, 341-372.

Leary, M.R., 1983. Social anxiousness: the construct and its measurement. J. Pers. Assess. 47, 66-75.

Liebowitz, M.R., Gorman, J.M., Fyer, A.J., Klein, D.F., 1985. Social phobia. Review of a neglected anxiety disorder. Arch. Gen. Psychiatry 42, 729-736.

Lorig, T.S., Elmes, D.G., Zald, D.H., Pardo, J.V., 1999. A computer-controlled olfactometer for fMRI and electrophysiological studies of olfaction. Behav. Res. Meth. Instrum. Comput. 31, 370-375.

Lundström, J.N., Goncalves, M., Esteves, F., Olsson, M.J., 2003a. Psychological effects of subthreshold exposure to the putative human pheromone 4,16-androstadien-3one. Horm. Behav. 44, 395-401.

Lundström, J.N., Hummel, T., Olsson, M.J., 2003b. Individual differences in sensitivity to the odor of 4, 16-androstadien-3-one. Chem. Senses 28, 643-650.

Lundström, J.N., Olsson, M.J., 2005. Subthreshold amounts of social odorant affect mood, but not behavior, in heterosexual women when tested by a male, but not a female experimenter. Biol. Psychol. 70, 197-204.

Marks, I.M., 1969. Fears and phobias. Academic Press, New York.

Milad, M.R., Rauch, S.L., 2007. The role of the orbitofrontal cortex in anxiety disorders Ann. NY Acad. Sci. 1121, 546-561.

Mujica-Parodi, L.R., Strey, H.H., Frederick, B., Savoy, R., Cox, D., Botanov, Y., Tolkunov, D., Rubin, D., Weber, J., 2009. Chemosensory cues to conspecific emotional stress activate amygdala in humans. PLoS ONE 4, e6415.

Pause, B.M., Ohrt, A., Prehn, A., Ferstl, R., 2004. Positive emotional priming of facial affect perception in females is diminished by chemosensory anxiety signals. Chem. Senses 29, 797-805.

Penn, D.J., Oberzaucher, E., Grammer, K., Fischer, G., Soini, H.A., Wiesler, D., Novotny, M.V., Dixon, S.J., Xu, Y., Brereton, R.G., 2007. Individual and gender fingerprints in human body odour. J. R. Soc. Interface 4, 331-340.

Plomin, R., Daniels, D., 1986. Genetics and shyness. In: Jones, W.H., Cheek, J.M., Briggs, S.R. (Eds.), Shyness: Perspectives on research and treatment. Plenum, New York, pp. 63-80.

Russell, M.J., 1976. Human olfactory communication. Nature 260, 520-522.

Savic, I., Berglund, H., Gulyas, B., Roland, P., 2001. Smelling of odorous sex hormone-like compounds causes sex-differentiated hypothalamic activations in humans. Neuron 31, 661-668.

Savic, I., Berglund, H., Lindstrom, P., 2005. Brain response to putative pheromones in homosexual men. Proc. Natl. Acad. Sci. USA 102, 7356-7361.

Sobel, N., Prabhakaran, V., Desmond, J.E., Glover, G., Sullivan, E.V., Gabrieli, J.D., 1997. A method for functional resonance imaging of olfaction. J. Neurosci. Meth. 78, $115-121$.

Stables, L.A., Kennan, R.P., Gore, J.C., 1998. Asymmetric spin-echo imaging of magnetically inhomogeneous systems: theory, experiment, and numerical studies. Magn. Reson. Med. 40, 432-442.

Stemberger, R.T., Turner, S.M., Beidel, D.C. Calhoun, K.S., 1995. Social phobia: an analysis of possible developmental factors. J. Abnorm. Psychol. 104, 526-531.

Stern, K., Mcclintock, M.K., 1998. Regulation of ovulation by human pheromones Nature 392, 177-179.

Talairach, J., Tournoux, P., 1988. Co-planar stereotaxic atlas of the human brain: 3 dimensional proportional system: an approach to cerebral imaging. Thieme Stuttgart, Germany.

Tillfors, M., Furmark, T., Marteinsdottir, I., Fischer, H., Pissiota, A., Langstrom, B. Fredrikson, M., 2001. Cerebral blood flow in subjects with social phobia during stressful speaking tasks: a pet study. Am. J. Psychiatry 158, 1220-1226.

Tsukatani, T., Miwa, T., Furukawa, M., Costanzo, R.M., 2003. Detection thresholds for phenyl ethyl alcohol using serial dilutions in different solvents. Chem. Senses 28 25-32.

Wyart, C., Webster, W.W., Chen, J.H., Wilson, S.R., Mcclary, A., Khan, R.M., Sobel, N. 2007. Smelling a single component of male sweat alters levels of cortisol in women. J. Neurosci. 27, 1261-1265.

Zhou, W., Chen, D., 2008. Encoding human sexual chemosensory cues in the orbitofrontal and fusiform cortices. J. Neurosci. 28, 14416-14421.

Zhou, W., Chen, D., 2009. Fear-related chemosignals modulate recognition of fear in ambiguous facial expressions. Psychol. Sci. 20, 177-183. 